\title{
Verbandmittel adäquat einsetzen
}

\author{
Effizient und wirtschaftlich Im Versorgungsprozess trägt die Wundauflage \\ nur wenig zum Heilungsfortschritt bei, dennoch gilt ihr oft die gesamte Auf- \\ merksamkeit. So ist bei stagnierenden Wundverhältnissen oder angesichts \\ keiner Fortschritte im Heilungsprozess häufig die erste Idee, von einem Produkt \\ auf ein anderes zu wechseln. Warum dieser Ansatz oft nicht zum Erfolg führt, \\ erläutert Wundexpertin Kerstin Protz.
}

I n Deutschland sind über 2,7 Millionen Menschen auf die Versorgung mit Verbandmitteln angewiesen. Davon haben ca. 900.000 eine chronische Wunde. Aufgrund des demographischen Wandels ist zu erwarten, dass diese Anzahl in den kommenden Jahren ansteigt. Neben gravierenden Einschränkungen in der Lebensqualität der Betroffenen bedeutet der hohe Bedarf an Verbandmitteln eine Kostenbelastung für das Gesundheitssystem.

\section{Verordnungs- und Erstattungsfähigkeit wird neu geregelt}

Bisher ist jedes Produkt, das in Deutschland als Verbandmittel zugelassen ist, verordnungs- und erstattungsfähig. Die verfügbare $\mathrm{Pa}$ lette umfasst weit über 7.000 Produkte, wenn man alle Hersteller, Größen und Re-Importe berücksichtigt. Angesichts dieser unüberschaubaren Vielfalt hat der Gesetzgeber mit dem „Gesetz für mehr Sicherheit in der Arzneimittelversorgung (GSAV)“ im August 2019 die Verbandmitteldefinition neu geregelt. In $\$ 31$, Abs. 1a SGB V steht: „Verbandmittel sind Gegenstände einschließlich Fixiermaterial, deren Hauptwirkung darin besteht, oberflächengeschädigte Körperteile zu bedecken, Körperflüssigkeiten von oberflächengeschädigten Körperteilen aufzusaugen oder beides zu erfüllen. Die Eigenschaft als Verbandmittel entfällt nicht, wenn ein Gegenstand ergänzend weitere Wirkungen entfaltet, die ohne pharmakologische, immunologische oder metabolische Wirkungsweise im menschlichen Körper der Wundheilung dienen, beispielsweise indem er eine Wunde feucht hält, reinigt, geruchsbindend, antimikrobiell oder metallbeschichtet ist." Zeitgleich erhielt der Gemeinsame Bundesausschuss (G-BA) den Auftrag, innerhalb eines Jahres verbindlich festzulegen, welche Verbandmittel zukünftig verordnungs- und erstattungsfähig sind. Sowohl auf Seiten der Hersteller als auch der Versorger bestehen große Befürchtungen, dass ein erheblicher Anteil der aktuellen Produkte zukünftig im Zuge dieser Neuregelung vom Patienten selbst zu zahlen ist. Zudem ist zu erwarten, dass die Krankenkassen ihrerseits den Gesetzestext inter- pretieren und möglicherweise bisher erstattete Verbandmittel nicht mehr als Teil des Leistungskataloges ansehen. Hierzu könnten Produkte zählen, die ausschließlich der Wundreinigung oder dem Hautschutz dienen.

\section{Kausaltherapie sichert den Heilungserfolg}

Verbandmittel gehören nicht zu den Hilfsmitteln. Daher ist für deren Verordnung die Markierung der Ziffer 7 für Hilfsmittel auf dem rosafarbenen Muster-16-Rezept zulasten der gesetzlichen Krankenversicherung (GKV) unzulässig. Derzeit gilt für Verbandmittel nicht die sogenannte Aut-idem-Regelung ( $\$ 73$ Abs. 5 SGB V). Somit darf die Apotheke nicht, wie bei Arzneimitteln, ein wirkstoffgleiches preisgünstigeres Produkt aus derselben Gruppe abgeben.

\section{- Eine erfolgreiche Wundbehandlung basiert primär auf einer adäquaten Diagnostik und der Einleitung einer darauf aufbauenden Kausaltherapie.}

Im Versorgungsprozess trägt die Wundauflage nur wenig zum Heilungsfortschritt bei, dennoch liegt der Fokus oft zu sehr darauf. So ist bei stagnierenden Wundverhältnissen oder angesichts keiner Fortschritte im Heilungsprozess häufig die erste Idee, von einem Produkt auf ein anderes zu wechseln. In der Konsequenz erhöhen sich die Materialkosten, während sich ungenutzte Verbandmittel beim Patienten ansammeln. Eine erfolgreiche Wundbehandlung basiert aber primär auf einer adäquaten Diagnostik und der Einleitung einer darauf aufbauenden Kausaltherapie:

- Nach dem Prinzip „ohne Strom kein Licht“ bedeutet dies für Wunden, die infolge einer arteriellen Durchblutungsstörung entstanden sind, dass zunächst Maßnahmen zur Revaskularisation ergriffen werden. 
_ Für die Abheilung eines Ulcus cruris venosum ist eine sachgerechte und konsequente Kompressionstherapie erforderlich.

- Bei einem Dekubitus liegt der Fokus auf einer Reduktion und Vermeidung von Druck-, Reibe- und Scherkräften.

- Die adäquate Druckentlastung ist, neben der Stoffwechseleinstellung, maßgeblich für die Behandlung eines diabetischen Fußulkus.

Diese Beispiele unterstreichen, dass die lokale Wundversorgung nur im Zusammenspiel mit einer entsprechenden Kausaltherapie erfolgreich sein kann. Die Wundversorgung orientiert sich im Anschluss an diversen Kriterien: zum Beispiel Patientenbedürfnissen und -akzeptanz, Schmerzen, Heilungsphase und Wundstadium, Infektanzeichen, Gerüchen, Exsudatmenge und -beschaffenheit, Wundrand und -umgebung, Kontinenzsituation, Handhabbarkeit und wirtschaftlichen Aspekten. Ergänzend fordert das Wirtschaftlichkeitsgebot des $\$ 12$ SGB V, dass Versorgungsmaßnahmen ,ausreichend, zweckmäßig und wirtschaftlich“ sein müssen und „das Maß des Notwendigen nicht überschreiten“ dürfen.

\section{Material sinnvoll und wirtschaftlich einsetzen}

Ein Verbandwechsel bedeutet für die meisten Patienten eine Stresssituation, die als Teil einer wiederkehrenden Routine wiederholt Belastungen mit sich bringt. Neben Schmerzen und weiterer Manipulation an der Wunde beeinflusst ein häufiger Verbandwechsel den Abheilungsprozess negativ, da die Wundruhe gestört wird. Die meisten modernen Wundauflagen sind darauf ausgelegt, bis zu sieben Tage auf der Wunde zu verbleiben. Die Wechselintervalle orientieren sich an Herstellerangaben, am Wundzustand und Exsudation. Bei nachfolgenden Wunden sind allerdings mindestens tägliche Verbandwechsel zu erwägen: zum Beispiel infizierte Wunden, venöse Beinulzerationen in der initialen Entstauungsphase, exulzerierende Tumorwunden und akute Verbrennungen.

Abseits von diesen Ausnahmen sollte ein Verbandwechsel nicht öfter als dreimal wöchentlich erfolgen. Zudem wird zwischen primären und sekundären Wundauflagen unterschieden. Primäre Wundauflagen liegen direkt auf dem Wundgrund auf. Hierzu gehören feinporige Polyurethanschaum-, Hydrokolloidverbände, Vlieskompressen mit Superabsorber. Tiefe und zerklüftete Wunden sind vorab mit Alginaten, Hydrofasern oder Cavity-Schäumen aufzufüllen. Sekundärverbände decken die primären Wundauflagen bzw. Wundfüller ab (z.B. semipermeable Transparentfolienverbände, (Saug-)Kompressen/Vlieskompressen mit Superabsorber oder feinporige Polyurethanschaumverbände). Sie können deren Funktion unterstützen und dienen als Schutz und Fixierung

\section{"Viel hilft viel“ - eine trügerische Annahme}

Wunden durchlaufen verschiedene Heilungsphasen: Reinigungs-, Granulationsphase und Epithelisierung. Daher ist die Verordnung derselben Verbandmittel über einen längeren Zeitraum generell nicht sinnvoll. Entsprechend sollten bei einer Überleitung aus dem klinischen in den ambulanten Bereich maximal zehn Wundauflagen verordnet werden. So kann auf individuelle Wundveränderungen flexibel reagiert werden.

Die sachgemäße Applikation der Verbandmittel ist die Voraussetzung dafür, dass sie ihre Funktion entsprechend erfüllen können. Das Übereinanderschichten von Wundauflagen ist hingegen

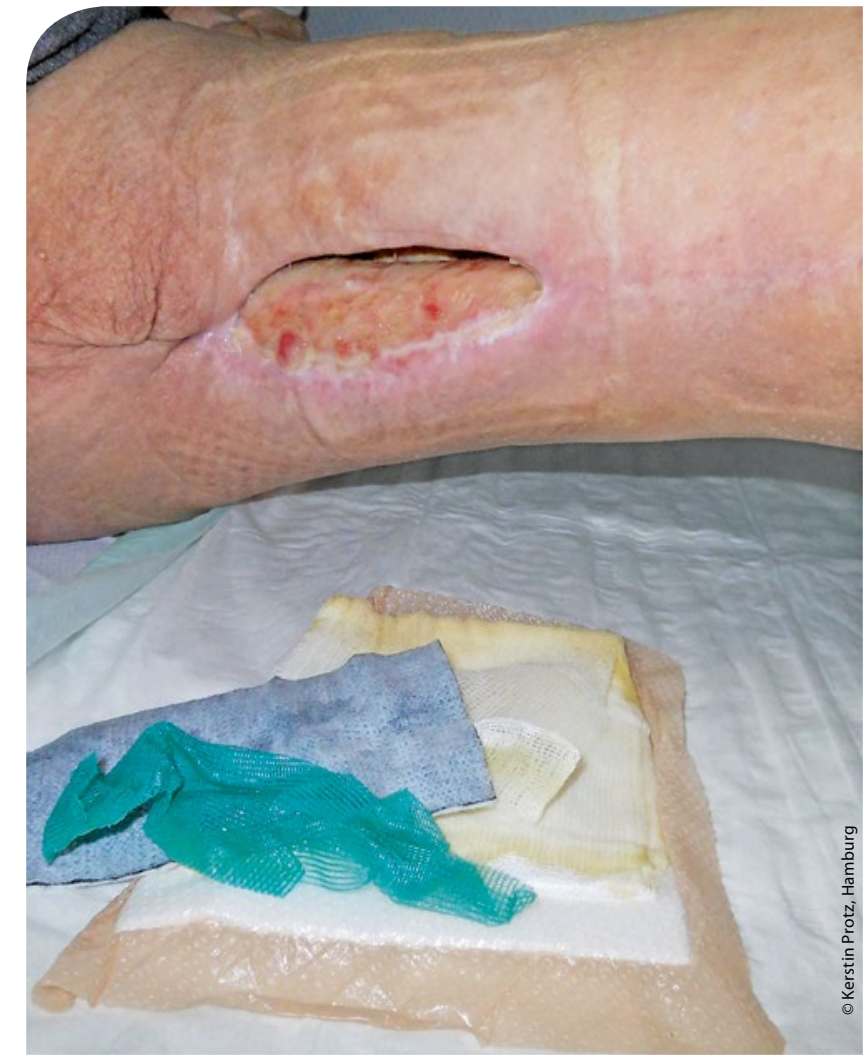

Abb. 1: Unwirtschaftliche Versorgung: „Wundburger" aus keimbindender Wundauflage, Aktivkohle, Kompressen und Schaumverband

nur selten sinnvoll und führt oft zu einem sogenannten „WundBurger" (Abb. 1). Dieser ist kostenintensiv und trägt dazu bei, dass sich die Wirkweise einzelner Produkte gegenseitig aufhebt. Als Faustregel sind daher zwei Wundauflagen, plus gegebenenfalls Fixiermaterial, ausreichend. Nachfolgende Produktanwendungen und -kombinationen kommen im Praxisalltag vor, dabei ist zu beachten:

„Die Wunde kann nicht lesen“: Dieses Motto erinnert an die Tatsache, dass eine Wundauflage nie mit der bedruckten Seite auf die Wunde appliziert wird (Abb. 2).

Keine täglichen Wechsel von Alginaten, Hydrofasern und Hydrogelen: In der autolytischen Wundreinigung werden diese Produkte genutzt, um Keime, Gewebereste und sonstige Fremdkörper sanft aus der Wunde zu entfernen. Aus wirtschaftlichen Gründen sind diese Produkte meist erst nach drei Tagen zu wechseln.

Hydrogele nicht mit saugenden Produkten abdecken: Ihre Feuchtigkeit wird durch solche Produkte, z.B. Alginate, Hydrofasser, feinporige Polyurethanschaumverbände, absorbiert.

Kein Einsatz von Hydrogelen bei stark exsudierenden Wunden: Diese führen zu einem zusätzlichen Feuchtigkeitsaufkommen sowie zu Mazerationen und erhöhen zudem das Infektionsrisiko. 


\section{PFLEGE KOLLEG}

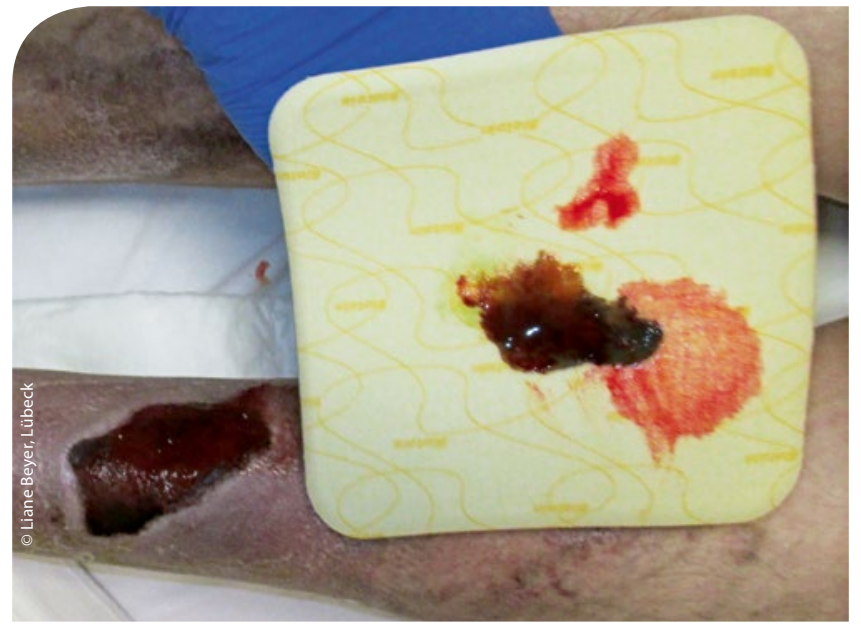

Abb. 2: Ein feinporiger Polyurethanschaumverband wurde falsch herum appliziert.

Hydrophobe Wundauflagen $\left(\right.$ Cutimed $^{\circledR}$ sorbact $^{\circledR}$ ) nicht mit Fettgazen oder folienbeschichteten Produkten kombinieren: Sie sind darauf ausgelegt, Keime in direktem Wundkontakt zu binden. Durch Folienverbände wird zudem ein feuchtes Wundklima erzeugt, das ein Keimwachstum fördert.

Alginat-Tamponaden nicht in kleinen Wundtaschen einsetzen: Da es sich um Einmalprodukte handelt und erhebliche Restmengen zu verwerfen sind, sind aus wirtschaftliche Gründen alternativ kleine Alginat-Kompressen (z.B. 5 x $5 \mathrm{~cm}$ ) zu nutzen.

Keime können nur einen Tod sterben: Antiinfektive Wundauflagen, beispielsweise mit Silber, Polihexanid (PHMB) oder hydrophobe Produkte, werden aus wirtschaftlichen Gründen nicht miteinander kombiniert.

Unter Kompressionsversorgungen nur Wundauflagen mit guten Retentionseigenschaften und ausreichendem Aufnahmevermögen einsetzen: Ansonsten wird das Exsudat durch den Kompressionsdruck unter der Auflage hervorgedrückt, das führt zu Hautmazerationen/-läsionen. Geeignet sind zum Beispiel Vlieskompressen mit Superabsorbern oder feinporige Polyurethanschaumverbände (gegebenenfalls mit Superabsorbern).

\section{- Für den Wechsel der Wundauflagen gilt die Faustregel: so häufig wie nötig und selten wie möglich.}

Für den Wechsel der Wundauflagen gilt die Faustregel: so häufig wie nötig und selten wie möglich. Verbandwechsel, die nur der Wundkontrolle und -beobachtung dienen sollen - etwa bei der Visite - bedeuten unnötigen Stress für Patient und Wunde. Alternativ sind bei entsprechenden Wundverhältnissen durchsichtige beziehungsweise transparente Produkte zu erwägen, beispielsweise
Folienverbände. Hierbei ist allerdings zu beachten, dass nicht jeder Patient seine Wunde sehen kann und möchte.

III

\section{PFLEGE EINFACH MACHEN}

Bei den modernen Wundauflagen handelt es sich in der Regel um hochspezialisierte Produkte, deren Einsatz einen gewissen Kenntnisstand voraussetzt. Leider wird eine sachgerechte Beratung durch das Gesundheitswesen nicht honoriert; Experten müssen entsprechende Leistungen ggf. durch den Verkauf von Wundauflagen finanzieren, was zu falschen Anreizen führen kann.

Nur wenn zeitgemäße Verbandmittel basierend auf Diagnostik und Kausaltherapie, zielgerichtet, entsprechend ihrer Wirkweise und orientiert an Wundzustand und -phase eingesetzt werden, können sie gleichzeitig effizient und wirtschaftlich sein.

Jeder Anwender sei dazu angeregt, die Herstellerangaben zu beachten, aktuelle Publikationen zu verfolgen und den Sachstand selbstständig zu recherchieren.

Die Literaturliste finden Sie im HEILBERUFE eMag auf springerpflege. de

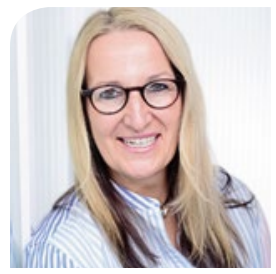

\section{Kerstin Protz}

Gesundheits- und Krankenpflegerin Projektmanagerin Wundforschung im Institut für Versorgungsforschung in der Dermatologie und bei Pflegeberufen (IVDP) am UKE, Vorstandsmitglied des Wundzentrum Hamburg kerstin.protz@gmx.de 